\title{
Deviations from cooperative growth mode during eutectoid transformation: insights from phase-field approach
}

\author{
Accepted in Acta Materialia on $6^{\text {th }}$ August, 2014
}

\author{
Kumar Ankit ${ }^{\mathrm{a}, \mathrm{b}, *}$, Rajdip Mukherjee ${ }^{\mathrm{a}, \mathrm{b}}$, Tobias Mittnacht $^{\mathrm{b}}$, Britta Nestler ${ }^{\mathrm{a}, \mathrm{b}}$ \\ ${ }^{a}$ Institute of Materials and Processes, Karlsruhe University of Applied Sciences, Moltkestr. 30, 76133 Karlsruhe, Germany \\ ${ }^{b}$ Institute of Applied Materials - Reliability of Components and Systems, Karlsruhe Institute of Technology, Haid-und-Neu-Str. 7, 76131 Karlsruhe, Germany
}

\begin{abstract}
The non-cooperative eutectoid transformation relies on the presence of pre-existing cementite particles in the parent austenitic phase and yields a product, popularly known as the divorced eutectoid. In isothermal conditions, two of the important parameters, which influence the transformation mechanism and determine the final morphology are undercooling (below $\mathrm{A}_{1}$ temperature) and interparticle spacing. Although, the criteria which governs the morphological transition from lamellar to divorced is experimentally well established, numerical studies that give a detailed exposition of the non-cooperative transformation mechanism, have not been reported extensively. In the present work, we employ a multiphase-field model, that uses the thermodynamic information from the CALPHAD database, to numerically simulate the pulling-away of the advancing ferrite-austenite interface from cementite, which results in a transition from lamellar to divorced eutectoid morphology in $\mathrm{Fe}-\mathrm{C}$ alloy. We also identify the onset of a concurrent growth and coarsening regime at small inter-particle spacing and low undercooling. We analyze the simulation results to unravel the essential physics behind this complex spacial and temporal evolution pathway and amend the existing criteria by constructing a Lamellar-Divorced-Coarsening (LDC) map.
\end{abstract}

Keywords: Non-cooperative growth, Divorced eutectoid transformation, Phase-field method, Coarsening

\section{Introduction}
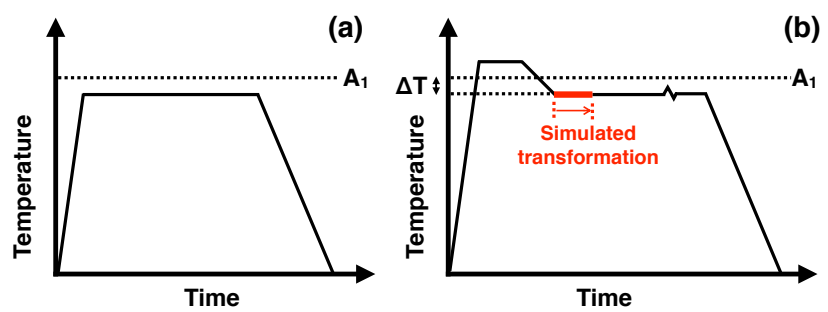

Figure 1: Typical spherodizing annealing heat treatment cycles [9]. (a) Subcritical annealing is carried out slightly below the $\mathrm{A}_{1}$ temperature and does not involve the formation of austenite. (b) Inter-critical annealing involves heating the hypereutectoid steel to fully austenise it, with a small amount of cementite remaining undissolved and then, holding it just below $\mathrm{A}_{1}$ temperature. The final transformation product is known as the divorced eutectoid. The divorced eutectoid transformation, that is numerically simulated in the present work (for three different undercoolings, $\Delta T$ ), is shown by the colored (thick) line.

The eutectoid transformation in steel involves the decomposition of the parent austenite $(\gamma)$ into two product phases, ferrite $(\alpha-\mathrm{Fe})$ and cementite $\left(\theta-\mathrm{Fe}_{3} \mathrm{C}\right)$. When both the product phases, evolve cooperatively, sharing a common growth front with austenite, the morphology of the resulting product is lamellar, popularly known as pearlite [1, 2, 3, 4]. On the

\footnotetext{
${ }^{*}$ Corresponding author. Tel.: +49 721 608-45022.

Email address: ankit.kumar2@hs-karlsruhe.de (Kumar Ankit)
}
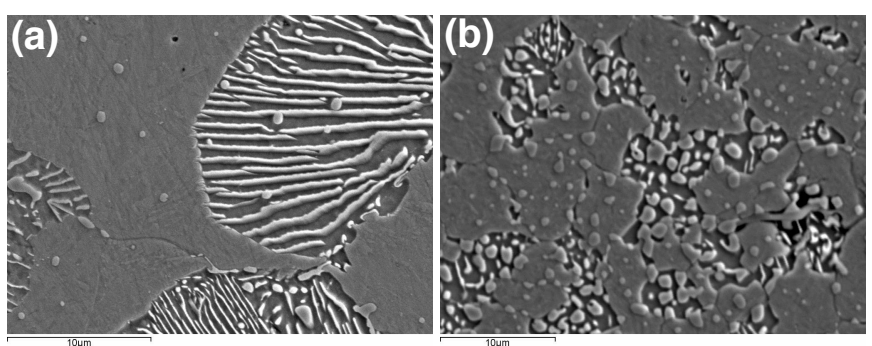

Figure 2: Cooperative and non-cooperative growth regimes are observed during eutectoid transformation in Fe-0.92C-0.66Si-1.58Mn-1.58Cr-0.12Ni-0.05Mo$0.178 \mathrm{Cu}$ (wt. \%) alloy. Samples are austenized at $870{ }^{\circ} \mathrm{C}$ for 2.5 hours and held for $\sim 65$ minutes below the eutectoid temperature (at $T_{1}$ and $T_{2}$ ). These are finally quenched to ambient temperature. (a) Lamellar and (b) divorced eutectoid morphologies are obtained for $\mathrm{T}_{1}=710^{\circ} \mathrm{C}$ and $\mathrm{T}_{2}=705^{\circ} \mathrm{C}$, respectively (private communication with Z.X. Yin and H.K.D.H. Bhadeshia),

contrary, under a given set of conditions (low undercooling and small inter-particle spacing of pre-exisiting cementite), the $\alpha / \gamma$ advancing transformation front begins to pull-away from cementite, leading to the formation of a divorced eutectoid. Hillert et al. [3] establish that a pearlitic colony comprises of inter-penetrating bi-crystals of ferrite and cementite phases. Steels with a fully pearlitic microstructure $(0.8 \mathrm{wt} . \% \mathrm{C})$, find extensive application in the manufacture of ropes, where high tensile strength is desirable.

Manufacture of a significant proportion of engineering com- 
ponents, obligate the use of steels with low hardness and good machinability (for e.g. in ball-bearings [5]). Two, well-known spherodizing annealing heat treatment cycles, that are adopted to soften the pearlite, prior to machining, are shown in Fig. 1 The sub-critical annealing involve the spherodization of the fine pearlite, by holding the hypoeutectoid steel isothermally, just below the $A_{1}$ temperature, as shown in Fig. 11a). The driving force for morphological transition is the reduction in $\theta / \alpha$ interfacial area. For softening hypereutectoid steels, intercritical annealing [Fig. 1,b)] is a more economical method (see [5] and references therein). The steel is fully austenised, such that a small amount of cementite particles remain undissolved, and then held below $\mathrm{A}_{1}$ to generate a spherodized transformation product (cementite particles embedded in ferritic phase), popularly known as divorced eutectoid microstructure, which is much softer than the lamellar counterpart i.e. pearlite [Fig. 2(a)]. Experimental studies indicate that the presence of preexisting cementite particles in the parent austenitic matrix results in the non-cooperation between the ferrite and cementite phases [6, 7, 8] and yields divorced eutectoid as the final transformation product [Fig. 2(b)].

The history of divorced eutectoid dates back to the time of Honda and Saito [10], who report the morphological dependence of the final-transformed product (lamellar to completely spehrodized) on the austenising temperature. Oyama et al. [6] describe a heat treatment schedule, that is adopted for spherodizing a microstructure, comprising of a mixture of pearlite and proeutectoid cementite. Verhoeven and Gibson [11] develop a theoretical framework (for binary $\mathrm{Fe}-\mathrm{C}$ alloy) to establish the criteria, that governs the transition from lamellar to divorced eutectoid morphology. Luzginova et. al [12] study the influence of chromium concentration on the formation of divorced pearlite in a hypereutectoid steel. Pandit and Bhadeshia [13] amend the earlier theory of lamellar to divorced eutectoid transition, by accounting for the diffusion of carbon along the transformation front.

It is apparent from the brief literature survey, that much of the investigation of divorced eutectoid transformation is primarily limited to experimental and theoretical studies. They delineate the basic concept of the evolution mechanism, but unable to provide the finer details required for tailoring the mictrostructure to achieve the desired properties (e.g. better machinability). Therefore, a theoretical understanding of the complex evolution pathways during the divorced eutectoid transformation is paramount to comprehend the final microstructure, which is indispensable from a technological point of view.

In view of establishing a synergy between theoretical and experimental studies concerning the eutectoid transformation, the phase-field method holds great promise in terms of the ability to describe the interface evolution in the diffusion length scale. In the present article, we use a multiphase-field model [14] to scrutinize (and amend) the existing theory by providing an in-depth understanding of the carbon redistribution mechanism, which has profound implications in eventual optimization of the process control parameters related to heat treatment of steel. Based on the insights from numerical simulations, our further intention is to depict the interplay between two important parameters
- (a) spacing between the pre-existing cementite particles and (b) undercooling, which can result in different eutectoid morphologies.

In the following section, the phase-field model, used for the present numerical simulations, is briefly outlined. The simulation results concerning the lamellar to divorced eutectoid transition and the concurrent growth and coarsening regime are discussed in subsections 3.1 and 3.2 respectively. In subsection 3.3. we summarize the presented simulation results by constructing a lamellar-divorced-coarsening (LDC) map. Section 4 concludes the article.

\section{Phase-field model}

The multiphase-field model is a common diffuse-interface approach for studying microstructural evolution accompanying phase transformations. The primary advantage of such a diffused-interface approach lies in the elegance with which it treats moving boundary problems by obviating the necessity to explicitly track the position of interfaces. In the present work, we use this approach for numerical simulations, which is coupled with CALPHAD database to study a binary Fe-C alloy system. The multiphase-field model equations, that are used in the present study, is briefly outlined in this section. The reader is referred to the previous studies [14, 15, 4, 16] for a more detailed description of the model equations and numerical methods.

The evolution of phases is governed by the phenomenological minimization of the grand potential functional $\Omega$,

$$
\begin{aligned}
& \Omega(T, \boldsymbol{\mu}, \boldsymbol{\phi})= \\
& \quad \int_{V}\left[\Psi(T, \boldsymbol{\mu}, \boldsymbol{\phi})+\left(\epsilon a(\boldsymbol{\phi}, \nabla \boldsymbol{\phi})+\frac{1}{\epsilon} w(\boldsymbol{\phi})\right)\right] d V,
\end{aligned}
$$

where $T$ is the temperature, $\boldsymbol{\mu}$ is the chemical potential vector comprising of $K-1$ independent chemical potentials, $\phi$ is the phase-field vector containing the volume fractions of the $\mathrm{N}$ phases and $\epsilon$ is the length scale related to the interface. $a(\phi, \nabla \phi)$ and $w(\phi)$ represent the gradient and obstacle potential type energy density, respectively and $V$ represents the domain volume. The grand potential density $\Psi(T, \boldsymbol{\mu}, \boldsymbol{\phi})$, which is the Legendre transform of the free energy density of the system $f(T, \boldsymbol{c}, \boldsymbol{\phi})$ is written as an interpolation of individual grand potential densities

$$
\begin{aligned}
& \Psi(T, \boldsymbol{\mu}, \boldsymbol{\phi})=\sum_{\alpha=1}^{N} \Psi_{\alpha}(T, \boldsymbol{\mu}) h_{\alpha}(\boldsymbol{\phi}) \\
& \Psi_{\alpha}(T, \boldsymbol{\mu})=f_{\alpha}\left(c^{\alpha}(T, \boldsymbol{\mu}), T\right)-\sum_{i=1}^{K-1} \mu_{i} c_{i}^{\alpha}(T, \boldsymbol{\mu}),
\end{aligned}
$$

where $h_{\alpha}(\boldsymbol{\phi})$ is an interpolation function of the form $h_{\alpha}(\boldsymbol{\phi})=$ $\phi_{\alpha}^{2}\left(3-2 \phi_{\alpha}\right)$. The evolution equation for the $\mathrm{N}$ phase-field variables can be written as,

$$
\begin{gathered}
\tau \epsilon \frac{\partial \phi_{\alpha}}{\partial t}=\epsilon\left(\nabla \cdot \frac{\partial a(\boldsymbol{\phi}, \nabla \boldsymbol{\phi})}{\partial \nabla \phi_{\alpha}}-\frac{\partial a(\boldsymbol{\phi}, \nabla \boldsymbol{\phi})}{\partial \phi_{\alpha}}\right) \\
-\frac{1}{\epsilon} \frac{\partial w(\boldsymbol{\phi})}{\partial \phi_{\alpha}}-\frac{\partial \Psi(T, \boldsymbol{\mu}, \boldsymbol{\phi})}{\partial \phi_{\alpha}}-\Lambda,
\end{gathered}
$$


where $\Lambda$ is the Lagrange parameter to maintain the constraint $\sum_{\alpha=1}^{N} \phi_{\alpha}=1$. The concentration fields are obtained by a mass conservation equation for each of the $K-1$ independent concentration variables $c_{i}$. The evolution equation for the concentration fields can be derived as,

$$
\begin{gathered}
\frac{\partial c_{i}}{\partial t}=\nabla \cdot\left(\sum_{j=1}^{K-1} M_{i j}(\phi) \nabla \mu_{j}\right) \\
M_{i j}(\boldsymbol{\phi})=\sum_{\alpha=1}^{N} M_{i j}^{\alpha} g_{\alpha}(\phi),
\end{gathered}
$$

where each $M_{i j}^{\alpha}$ represents the mobility matrix of the phase $\alpha$ (related to the diffusivity). The function $g_{\alpha}(\phi)$ is in general not the same as $h_{\alpha}(\phi)$ which interpolates the grand potentials, however, in the present description, we utilize the same. The thermodynamic data-fitting procedure to approximate the variation of the grand-potential of the respective phases as a function of chemical potential and the relation of the numerical simulation parameters with the corresponding quantities in the sharpinterface limit, are explained in the previous work [4].

\section{Results and Discussion}

\subsection{Lamellar to Divorced transition}

As we are primarily interested in amending the criteria which determines whether the eutectoid transformation front evolves by a cooperative (lamellar growth, which leads to the formation of pearlite) or a non-cooperative mechanism (resulting in divorced eutectoid), we use the same input parameters (volume diffusion constants and surface energies) for the present phasefield simulations that was used earlier by Ankit et al. [4] to simulate a pearlitic morphology. In order to account for the role of diffusion of carbon along the transformation front simultaneously, the interface diffusion constant is assumed to be 1000 times greater than volume diffusion constant in ferrite. The interface relaxation coefficient is derived from a thin-interface analysis which is described elsewhere [17, 14].

We study the temporal evolution of austenite, ferrite and cementite phases which is governed by the initial particle spacing at intercritical temperature and the undercooling below the eutectoid temperature $\left(A_{1}\right)$. The simulation domain width in the transverse direction directly controls the spacing (represented by $\lambda$ ) while the radius of the particles is kept same for consistency of the numerical results. In order to compare the present phase-field results with the classical theories, which introduce a criteria for lamellar to divorced transition based on experimental findings [11, 5], we limit the present discussion to a symmetric arrangement of pre-existing cementite particles which are embedded in an austenite matrix. The undercooling below the eutectoid temperature $(\Delta T)$ as well the particle spacing $(\lambda)$ is varied to study their effect on the resulting microstructure.

Fig. 3(a) shows the dependence of undercooling and particle spacing in stimulating a transition from lamellar to divorced morphology. It is noteworthy, that the numerical results accentuate the experimental findings which emphasize a greater
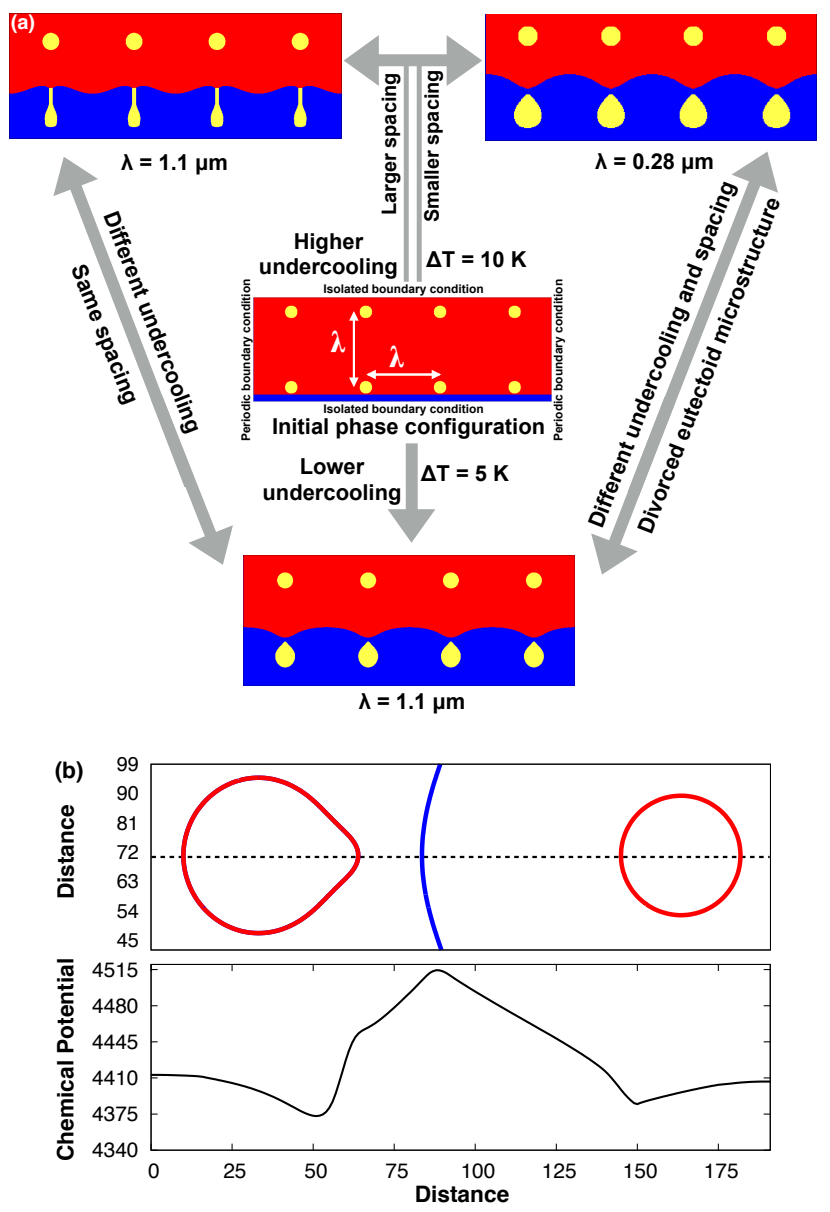

Figure 3: (a) Numerically simulated microstructures at two different undercoolings below the eutectoid temperature $(\Delta T=5$ and $10 \mathrm{~K})$ and particle spacing $(\lambda=0.28$ and $1.1 \mu \mathrm{m})$ starting from the same initial arrangement of the phases. The diagram shows that a cooperative growth regime is favored at higher undercooling and spacing leading to the formation of pearlitic lamellae. At lower undercoolings and smaller particle spacings, a non-cooperative mechanism predominates which results in the formation of a divorced eutectoid microstructure. (b) 1 -D chemical potential profile for $\Delta T=5 \mathrm{~K}$ and $\lambda=0.58 \mu \mathrm{m}$ plotted along the dashed line connecting the center of both the cementite particles. The profile shows that the carbon partitioned at the $\alpha / \gamma$ transformation front is incorporated by both the particles which results in non-cooperative eutectoid transformation.

tendency of the ferrite-austenite interface to pull away from cementite particles at low undercooling and small spacing. On the contrary, at larger spacing and higher undercooling, a cooperative growth regime is favored which results in the formation of pearlitic lamellae. On analyzing the simulated chemical potential profile in 1-D as shown in fig. 3(b), it is apparent that a divorced morphology forms due to the incorporation of partitioned carbon (at the advancing $\alpha / \gamma$ transformation front) into the existing cementite particles. Thus, a near overlap of the present simulation results with the existing theory demonstrate the general capability of phase-field method in capturing the topological changes during eutectoid transformation.

Fig. 4 compares the temporal evolution of the numerically simulated isolines corresponding to interphase interfaces for a lamellar growth [Fig. 4(a)] and divorced eutectoid [Fig. 4(b)], starting from a symmetric arrangement of cementite particles. 

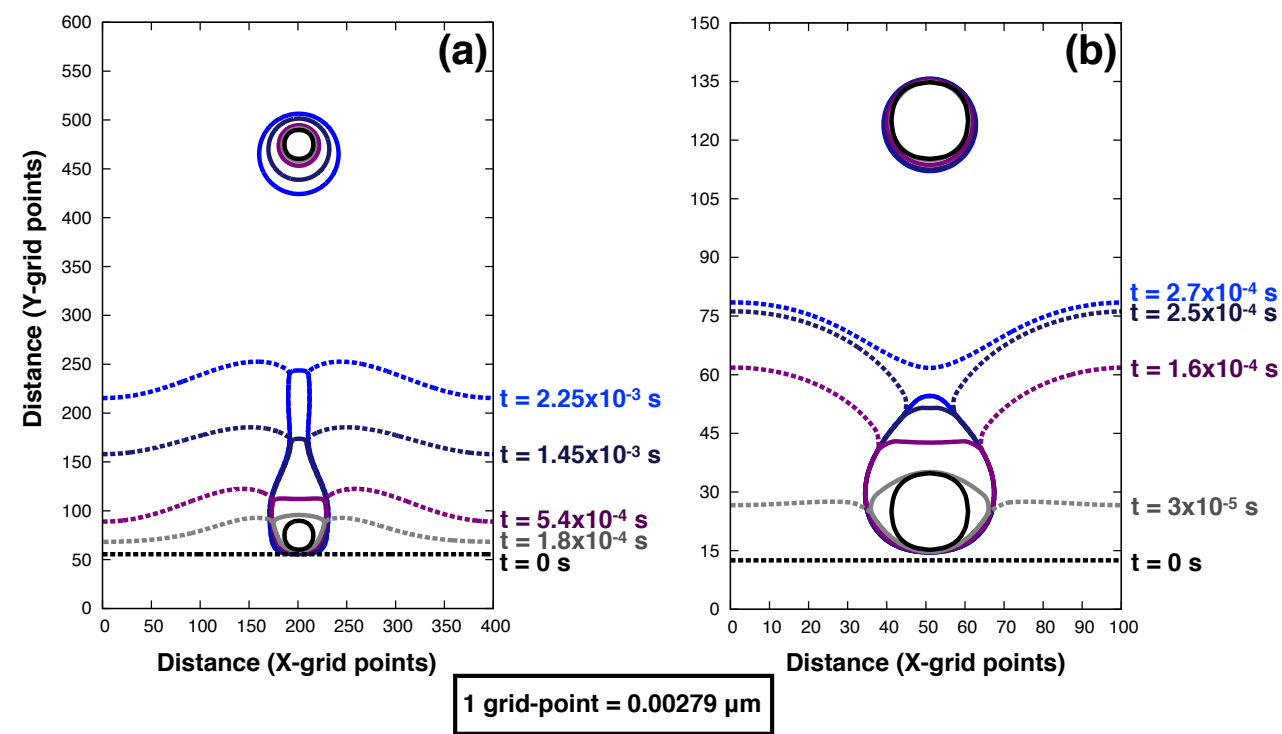

Figure 4: Temporal evolution of the isolevels $\phi_{\alpha}=0.5$ (dashed lines representing $\alpha / \gamma$ interface) and $\phi_{\theta}=0.5$ (solid lines representing $\theta / \gamma$ and $\theta / \alpha$ interfaces) for the (a) cooperative (resulting in pearlitic lamella) and (b) non-cooperative (resulting in divorced eutectoid) regimes. The pulling-away of the advancing ferrite-austenite interface is evident from the numerically simulated isolevels shown in (b). A comparison of the temporal evolution of the isolines in (a) and (b) indicate that the initial particle spacing ' $\lambda$ ' (number of grid-points along $\mathrm{X}$-axis) governs the switch between both the evolution regimes (for constant undercooling $\Delta \mathrm{T}=10 \mathrm{~K}$ ).

As the undercooling is constant for both the cases $(\Delta \mathrm{T}=10 \mathrm{~K})$, the evolution mode (cooperative or non-cooperative) is determined by the initial particle spacing ' $\lambda$ ' (represented by $\mathrm{X}$-axes in Fig. 4). At a lower value of ' $\lambda$ ' $(0.27 \mu \mathrm{m})$, the $\alpha / \gamma$ interface pulls-away from $\theta$, more commonly known as, the noncooperative growth. However, at a larger value of ' $\lambda$ ' $(1.11 \mu \mathrm{m})$, the growing phases, $\alpha$ and $\theta$ maintain a common transformation front, by evolving cooperatively.

\subsection{Concurrent growth and coarsening}

The most phenomenal finding of the present numerical studies is the isolation of concurrent growth and coarsening regime during eutectoid transformation. Fig. 5 shows the temporal evolution of phase contours which are overlaid on the chemical potential map, when the initial cementite spacing is reduced to $0.294 \mu \mathrm{m}$ at an undercooling of $5 \mathrm{~K}$ below the $A_{1}$ (eutectoid) temperature. An intermittent coarsening regime sets in before the pulling away of the $\alpha / \gamma$ interface from cementite particles. In order to provide a detailed exposition of this newly identified regime (which is not clearly visible in Fig. 5), we plot both the phase contours separately [in Fig. 6(a)] as well as the 1-D chemical potential profile along the dashed-line [in Fig. 6.b)] for different simulation time-steps. Depending on the initial distance from the ferrite-austenite transformation front, the cementite particles are labeled as 1 and 2. On comparing the 1-D chemical potential profiles for two different simulation timesteps $\left(t_{1}\right.$ and $\left.t_{2}\right)$, we find that a change in the carbon redistribution mechanism is stimulated which leads to coarsening of particles prior to the divorce from the growth front.

To begin with, the $\alpha / \gamma$ transformation front advances and forms an interface with the adjacent cementite particle 1 . As a result of this interaction, particle 1 starts to grow due to the incorporation of partitioned carbon primarily via the interface (transformation front) diffusion flux. It is noteworthy, that the particle 1 which shares a common interface with ferrite experiences a greater influx of partitioned carbon as compared to particle 2 , since the interface diffusivity is assumed to be 1000 times faster than the diffusion in austenite in all the present cases. As the diffusion fields of both the cementite particles overlap, particle 1 grows while the particle 2 shrinks, as shown in Figs. 6(a) and 6.c). At this stage, the driving force for coarsening predominates over the growth. The same is also reflected [Fig. 6.a)] by a temporal increase in the curvature of $\theta / \gamma$ interface of particle 1 which slowly approaches infinity and subsequently curves inwards. An advancement of $\alpha / \gamma$ transformation front towards particle 2 causes a shift in the carbon redistribution mechanism again; the driving force for cementite growth exceeds coarsening. We attribute a reduction in the distance between $\alpha / \gamma$ transformation front and particle 2 which makes the incorporation of partitioned carbon feasible at smaller distances via bulk diffusion flux. As a result, the chemical potential near the advancing $\alpha / \gamma$ front ascends leading to the growth of particle 2. This change in the carbon redistribution mechanism which results in predominance of growth over a coarsening regime is evident from the 1-D plot shown in Fig. 6(b).

It is worth clarifying that the "concurrent growth and coarsening" regime (denoted by ' $C$ ') reported in the present work principally differs from the particle coarsening in alloys which has been extensively reported in the literature [18, 19, 20, 21, 22]. Although, the reported regime ' $C$ ' does involve curvature driven coarsening of particles, the primary difference with the phenomena of conventional coarsening is attributed to the energetics of $\alpha / \theta / \gamma$ phase triple-junction which determines if the transformation proceeds by a cooperative (to yield lamellar pearlite) or by a non-cooperative regime (yielding divorced eutectoid). Further, the accompanying eutectoid transformation 


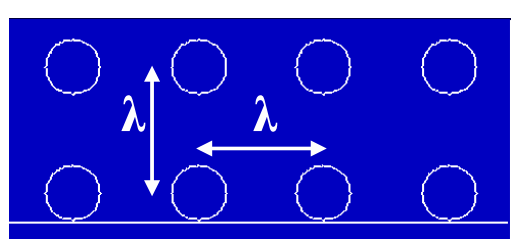

$\mathbf{t}=\mathbf{0 ~ s e c}$

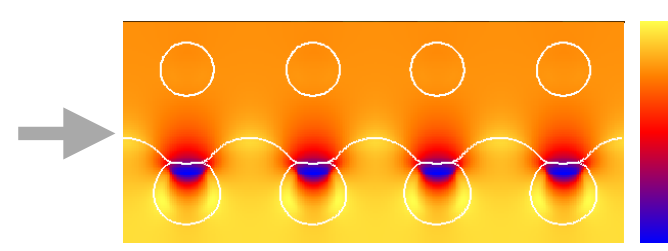

$\mathrm{t}=5.76 \times 10^{-4} \mathrm{sec}$

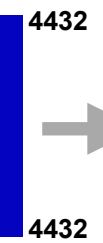

4432

4416.73

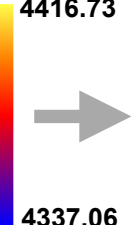

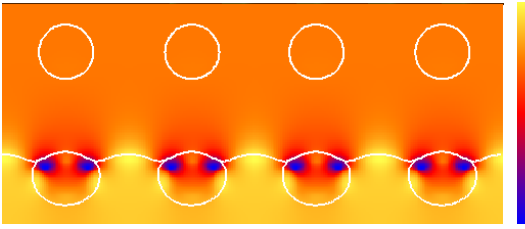

$t=1.11 \times 10^{-4} \mathrm{sec}$

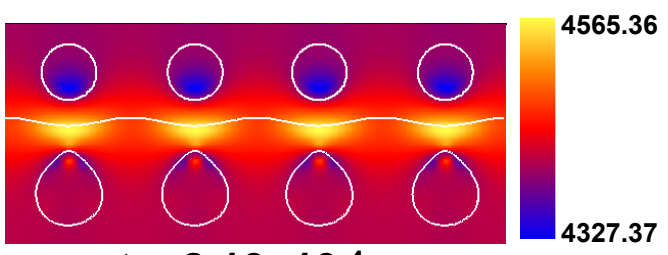

$\mathrm{t}=8.13 \times 10^{-4} \mathrm{sec}$

Figure 5: Temporal evolution of the phase contours which are plotted over the corresponding chemical potential maps during concurrent growth and coarsening regime $(\Delta T=7.5 \mathrm{~K}$ and $\lambda=0.294 \mu \mathrm{m})$. Coarsening can be observed clearly in Fig. 6
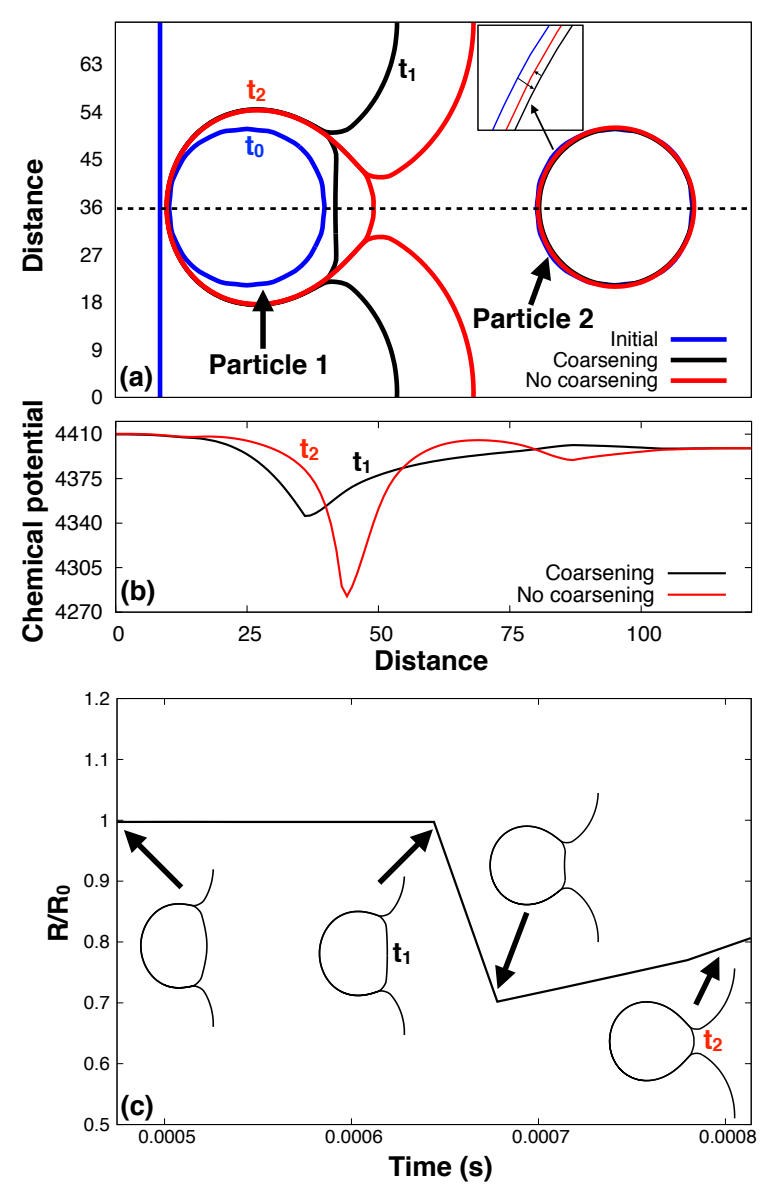

Figure 6: (a) Phase contours showing the subsequent shrinkage and growth of particle 2 as the $\alpha / \gamma$ front advances. (b) 1-D chemical potential profiles plotted along the dashed line in (a) which shows a deviation in the carbon redistribution mechanism during temporal evolution (as seen at $t_{1}=6.44 \times 10^{-4}$ seconds and $t_{2}=7.79 \times 10^{-4}$ seconds). (c) Temporal evolution of the scaled radius $\left(R / R_{0}\right)$ of particle 2 illustrating sharp deviations in the trend. The corresponding contours of particle 1 are also plotted along side at different time-steps which explains how the temporal change in curvature of $\theta / \gamma$ interface of particle 1 results in the onset of growth and coarsening regimes respectively. modifies the effective curvature of $\theta / \gamma$ interface of particle ' $A$ ' which increases the rate of coarsening as depicted by a decline in normalized radius of particle 'B' shown in Fig. 6. b). It can be argued that the reported regime holds a close resemblance with the discontinuous coarsening of grain boundary precipitates which could result in the formation of precipitate free zone (PFZ) along prior austenite grain boundaries [23]. However, on a careful examination, it is apparent that the physics of temporally evolving interphase interfaces which is reported in the present study is not only different, but also more complex when compared to the grain boundary interfaces involved in discontinuous coarsening.

\subsection{Lamellar-Divorced-Coarsening map (LDC)}

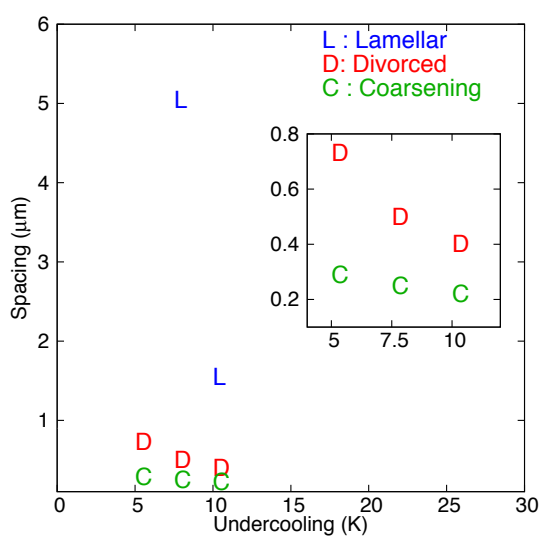

Figure 7: A morphological transition map showing the predominance of lamellar (L), divorced (D) and concurrent growth and coarsening (C) modes during the eutectoid transformation in Fe-C alloy system. The initial spacing between the cementite particles (at the intercritical temperature) as well as the undercooling below the $A_{1}$ temperature govern the switching among the three numerically simulated modes.

Having numerically simulated and comprehended the ipseity of the concurrent growth and coarsening regime which pre- 
cedes the non-cooperative eutectoid transformation, we construct a Lamellar-Divorced-Coarsening (LDC) transition map as shown in Fig. 7 to summarize the parametric study. The LDC transition map generated by conducting phase-field simulations for three different undercoolings $(5,7.5$ and $10 \mathrm{~K})$ below the eutectoid temperature and initial particle spacings predicts the morphology that is favored for a given set of initial conditions during an isothermal transformation. In a nut-shell, the most significant contribution of the work presented in the current letter is the addition of an alphabet ' $\mathrm{C}$ ' (acronym for concurrent growth and coarsening regime which is favored at smaller spacing and lower undercooling) to the classical Lamellar-Divorced (LD) map [11, 13]. Further, the present numerical findings are also in complete agreement with the existing theory for the divorced to lamellar morphological transition; lamellar morphology being more favorable at large spacings and high undercooling.

\section{Conclusions}

In conclusion, the spacing of the cementite particles embedded in the austenite matrix as well as undercooling below the eutectoid temperature entirely determines the final microtructure. An in-depth phase-field study of the isothermal transformation presented in this article, aids in selection of parameters to tailor the eutectoid microstructure appropriately. The present approach also captures the important transition between lamellar and divorced morphologies and sheds light on the change in carbon redistribution mechanism which is primarily governed by initial configuration of the phases. The concurrent growth and coarsening regime is identified for the first time which may be fundamentally difficult to isolate in experiments. Thus, the present numerical studies provide new insights into the transformation mechanism and amend the classical model of eutectoid transformation.

In future, it will be interesting to study the influence of asymmetrical arrangement of cementite particles on the final eutectoid morphologies. Large-scale numerical studies of the divorced eutectoid transformation for a random distribution of particles needs to be conducted to facilitate a direct comparison with the experimental microstructures.

\section{Acknowledgements}

The authors thank Z.X. Yin and Prof. H.K.D.H. Bhadeshia for the contribution of experimental microstructure and Prof. A. Choudhury for preliminary discussions. KA, RM and BN acknowledge the financial support of DFG in the framework of Graduate School-1483.

\section{References}

[1] Zener C. Kinetics of the Decomposition of Austenite. Wiley, NY, 1947.

[2] Hillert M. Jernkont Ann 1957;147:757.

[3] Hillert M. Decomposition of Austenite by Diffusional Processes. Interscience Publishers, New York, 1962.
[4] Ankit K, Choudhury A, Qin C, Schulz S, McDaniel M, Nestler B. Acta Mater 2013;61:4245.

[5] Bhadeshia H. Prog Mater Sci 2012;57:268 .

[6] Oyama T, Sherby O, Wadsworth J, Walser B. Scripta Metall Mater 1984; 18:799.

[7] Syn C, Lesuer D, Sherby O. Metall Mater Trans A 1994;25:1481.

[8] Taleff EM, Syn CK, Lesuer DR, Sherby OD. Metall Mater Trans A 1996; 27A:111.

[9] O’Brien J, Hosford W. J Mater Eng Perform 1997;6:69.

[10] Honda K, Saito S. J Iron Steel Inst 1920;102:261.

[11] Verhoeven J, Gibson E. Metall Mater Trans A 1998;29:1181.

[12] Luzginova N, Zhao L, Sietsma J. Metall Mater Trans A 2008;39 A:513.

[13] Pandit A, Bhadeshia H. P Roy Soc A-Math Phy 2012;468:2767.

[14] Choudhury A, Nestler B. Phys Rev E 2012;85:021602.

[15] Molnar D, Mukherjee R, Choudhury A, Mora A, Binkele P, Selzer M, Nestler B, Schmauder S. Acta Mater 2012;60:6961 .

[16] Mukherjee R, Choudhury A, Nestler B. Model Simul Mater Sc 2013; 21:075012.

[17] Karma A, Rappel WJ. Phys Rev E 1996;53:R3017.

[18] Mendoza R, Savin I, Thornton K, Voorhees P. Nat Mater 2004;3:385.

[19] Wu D. Nat Mater 2004;3:353.

[20] Ardell A, Ozolins V. Nat Mater 2005;4:309.

[21] Clouet E, Laé L, Épicier T, Lefebvre W, Nastar M, Deschamps A. Nat Mater 2006;5:482.

[22] Hoyt J. Nat Mater 2011;10:652.

[23] Ahmadabadi M, Shirazi H, Ghasemi-Nanesa H, Nedjad S, Poorganji B, Furuhara T. Mater Design 2011;32:3526. 